\title{
Implementación de sensores en los sistemas de riego automatizado
}

\section{Implementation of sensors in automated irrigation systems}

\section{Yenier Valencia Villegas}

Ingeniero en mecatrónica, Esp. Educación Superior a Distancia Universidad Nacional Abierta y a Distancia

yenier.valencia@unad.edu.co

\section{Yulian Sepúlveda Casadiego}

Ingeniero de Sistemas, Magister de Profundización en Educación con Enfasis en Educación Virtual

Universidad Nacional Abierta y a Distancia

yulian.casadiego@unad.edu.co

\section{RESUMEN.}

En la actualidad, los sensores agrícolas y ambientales son ampliamente utilizados en diversos proyectos de investigación que se llevan a cabo en la Universidad Nacional Abierta y a Distancia UNAD, especialmente en los proyectos de agricultura moderna que buscan fortalecer los procesos productivos de los pequeños y medianos agricultores. Son diversos parámetros que se pueden medir y controlar en un cultivo que, mediante una gestión adecuada, permiten realizar un uso eficiente del riego, fertilización y control de enfermedades, que se ve reflejado en un aumento de la productividad y calidad de los cultivos con menores costos de producción por el uso eficiente de los insumos agrícolas y reducción de costos de mano de obra en los sistemas automatizados. La adopción de tecnología en el sector agrícola colombiano no es tarea fácil, el desconocimiento y el costo de los mismos hace que sea un beneficio que habitualmente solo grandes productores pueden implementar en sus procesos productivos. Con una población mundial creciente, se hace indispensable que los pequeños y medianos agricultores se adapten a las nuevas tendencias tecnológicas que les permita suplir la demanda de alimentos cada vez más creciente, haciendo un uso racional de los recursos naturales y contribuyendo a reducir los impactos negativos en el cambio climático derivado de las malas prácticas agrícolas.

DOI: https://doi.org/10.22490/ECAPMA.3417 


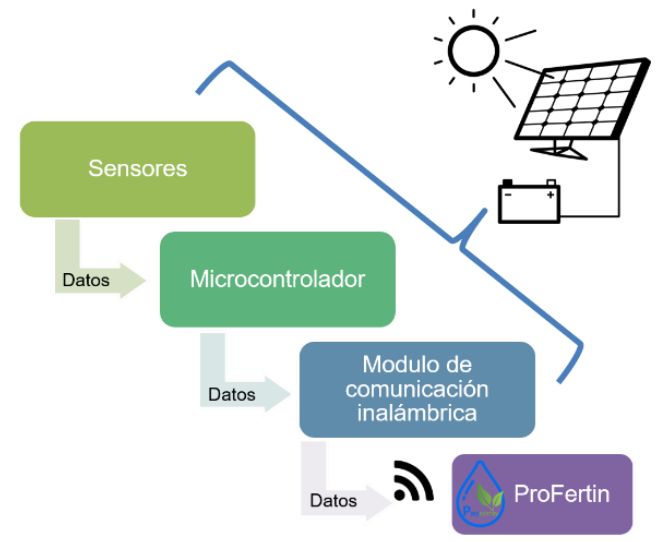

Figura 1. Módulos de sensores en sistema de riego automatizado

Palabras Clave: Sensores agrícolas; sistemas de riego; calidad de cultivos.

\begin{abstract}
.
At present, agricultural and environmental sensors are widely used in various research projects carried out at the Universidad Nacional Abierta y a Distancia UNAD, especially in modern agriculture projects that seek to strengthen the productive processes of small and medium farmers. There are various parameters that can be measured and controlled in a crop that, through proper management, allows efficient use of irrigation, fertilization and disease control, which is reflected in an increase in the productivity and quality of crops with lower production costs for the efficient use of agricultural inputs and reduction of labor costs in automated systems. The adoption of technology in the Colombian agricultural sector is not an easy task, ignorance and the cost of them makes it a benefit that only large producers can usually implement in their production processes. With a growing world population, it is essential that small and medium farmers adapt to new technological trends that allow them to meet the growing demand for food, making rational use of natural resources and contributing to reduce the negative impacts on climate change derived from bad agricultural practices.
\end{abstract}

Keywords: Agricultural sensors; irrigation systems; crop quality 


\section{INTRODUCCIÓN.}

"Entre las grandes tendencias de consumo en el mundo, el mercado agrícola presenta nuevas oportunidades de negocios alrededor de bioinsumos, agricultura sostenible, bioremediación de suelos y aguas, reproducción in vitro, semillas mejoradas con biotecnologías de punta" (Montenegro Gómez \& Hernández Ossa, 2015, p.100). Oportunidades que surgen del reto permanente que enfrenta el sector agrícola por la creciente demanda de la población mundial, que durante mucho tiempo ha intentado suplir aumentando las áreas cultivadas; sin embargo, la agricultura moderna permite aumentar el rendimiento de los cultivos, con la implantación del concepto de la agricultura de presión, como conjunto de técnicas orientadas a la optimización de las labores agrícolas tradicionalmente realizadas.

A raíz del incremento de los períodos secos producidos por el cambio climático en diferentes países del mundo, se debe implementar una nueva visión en la gestión del agua en la agricultura, implantado políticas públicas que permitan cuidar el recurso hídrico como fuente fundamental de vida, y es que "las problemáticas asociadas al recurso hídrico en el sector agropecuario están relacionadas con su manejo y las condiciones técnicas del cultivo, las cuales van a mitigar o a favorecer los impactos negativos" (Corredor Camargo, Fonseca Carreño, \& Páez Barón, 2015, p.80). Es en estos aspectos donde las tecnologías pueden tener un impacto positivo haciendo un uso adecuado de los recursos naturales como el agua; a través de sensores de humedad en el suelo, que permiten monitorear en tiempo real las necesidades de la planta y a su vez suministrar lo que la planta requiere en el momento justo. Otro tipo de sensor ampliamente utilizado es el sensor de Conductividad eléctrica (CE), que determina las necesidades nutricionales de la planta y a través de sistemas automatizados entregar la cantidad exacta de fertilizante que la planta requiere, evitando filtraciones de fertilizantes a las fuentes hídricas remediando su contaminación.

En este sentido, la implantación de estas tecnologías no solo permite monitorizar los parámetros del cultivo, además permite predecir y optimizar la gestión del cultivo en tiempo real; a través de los controladores de riego que reciben la información de los sensores instalados en todo el cultivo, que son los encargados de capturar la información de los parámetros que tienen incidencia sobre la planta como lo son la temperatura y humedad relativa del aire, temperatura del suelo, tensión del suelo, contenido volumétrico de agua (VWC), CE, alcalinidad 
o acidez de una solución $(\mathrm{pH})$, oxígeno disuelto, entre otros. La transferencia de la información de los sensores hacia el controlador se puede realizar vía comunicación inalámbrica (Radio frecuencia) o vía cableada (Voltaje). Sin embargo, "Los bajos niveles de escolaridad de campesinos hacen que haya desconocimiento de propuestas técnicas en la agricultura; además de la escasez de información y conocimientos sobre experiencias en condiciones específicas" (Mendoza Velásquez, Cano Muñoz, \& Rojas Sánchez, 2015, p.271).

En el Eje cafetero, la UNAD a través de su Centro de Investigación de Agricultura y Biotecnología (CIAB) y con el apoyo Tecnoparque Nodo Pereira del Servicio Nacional de Aprendizaje (SENA), han desarrollado e implantado tecnologías a bajo costo para el sector agrícola, que actualmente permiten que agricultores de la zona cuenten con estas tecnologías que les permita incrementar la rentabilidad de sus predios.

\section{DESARROLLO DEL TEMA}

\section{Riego por goteo}

Uno de los sistemas de riego más utilizado por su eficiencia y ahorro del agua es el riego por goteo localizado, una tecnología útil que conduce agua mediante una red de tuberías y es suministrada a la planta a través de emisores localizados en la zona radicular de la planta (Figura 2), "desde el punto de vista agronómico, se denominan riegos localizados porque humedecen un sector de volumen de suelo, suficiente para un buen desarrollo del cultivo" (Liotta, Carrión, Ciancaglini, \& Olguín, 2015, p.5). Para la implantación de un sistema de riego por goteo localizado automatizado, se requieren una serie de componentes básicos para su óptima operación; como primer elemento se debe contar con una fuente de abastecimiento de agua apta para el cultivo, el sistema hidráulico está compuesto por un equipo de bombeo, sistema de filtración, unidad de fertilización, tubería de conducción y emisores. Para la automatización del sistema se requieren los instrumentos de control (Motobomba, electroválvulas e inyectores de fertilizante) y medición ( $\mathrm{VWC}, \mathrm{pH}, \mathrm{CE}$, temperatura, entre otros) que son administrados por un equipo electrónico. 


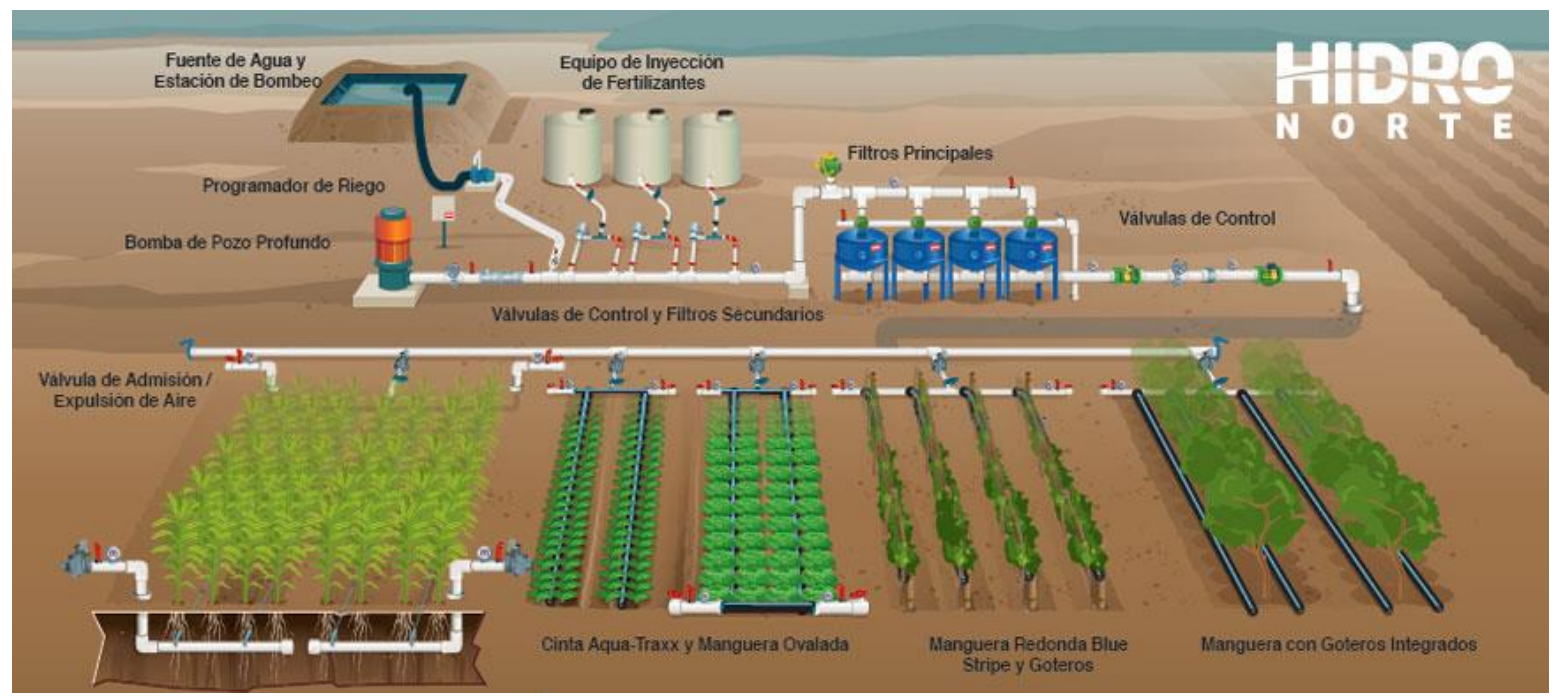

Figura 2. Esquema de sistema de riego por goteo. Fuente: Hidro Norte

El suministro de nutrientes para el cultivo en sus diferentes etapas de crecimiento se realiza mediante un sistema de fertirrigación, encargado de enviar los nutrientes a través del riego por goteo. "Para realizar la dosificación de fertilizantes se implementa un inyector de fertilizantes tipo Venturi, una pieza en forma de T con un estrechamiento que acelera la velocidad del agua provocando una depresión que succiona la solución fertilizante, inyectándola a la tubería" (Liotta, Carrión, Ciancaglini, \& Olguín, 2015, p.15). La instalación del inyector Venturi se realiza en el en paralelo a la electroválvula principal de riego (Figura 3), de esta manera se puede realizar el control determinado por las necesidades del cultivo, realizando la aplicación del agua con o sin inyección de fertilizante.

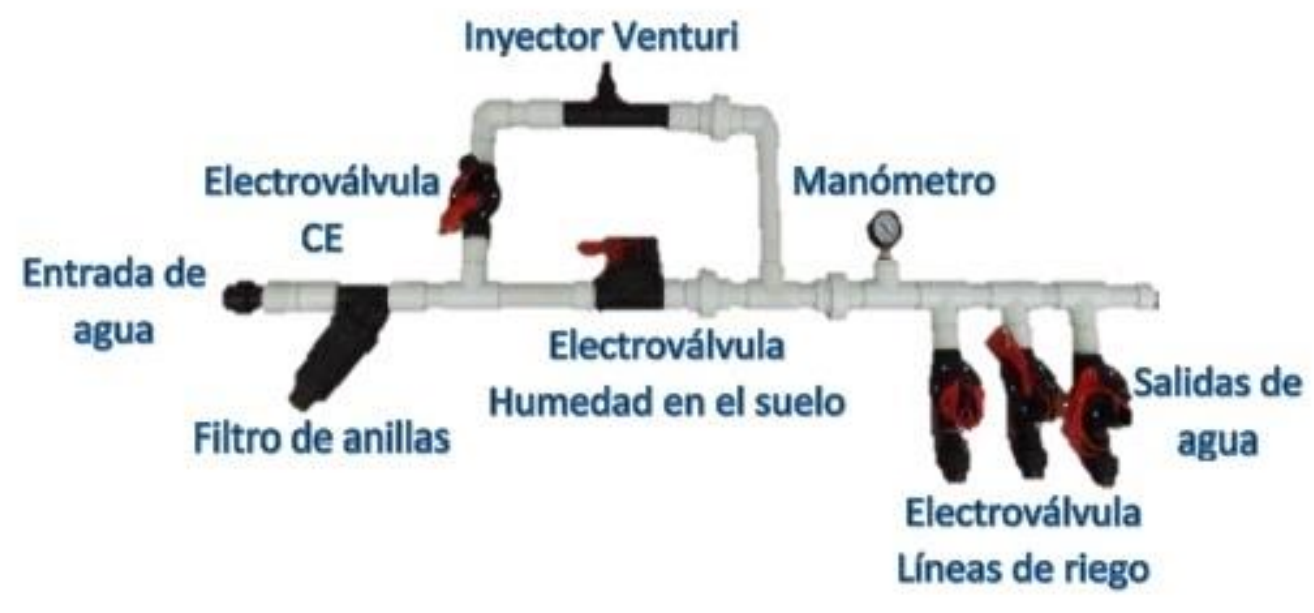

Figura 3. Esquema sistema hidráulico para el control del riego y fertilización 


\section{Automatización del riego por goteo}

La automatización del sistema de riego se realiza mediante un prototipo desarrollado en la UNAD denominado "ProFertin", un sistema inteligente que mediante sus algoritmos de control permite suministrar las cantidades de agua y fertilizante que la planta requiere de manera automática. ProFertin tiene una capacidad para la conexión de 12 sectores de riego, los cuales se pueden programar individualmente a través de los parámetros de configuración (Tabla 1), ajustando dichos parámetros a las necesidades de cada cultivo o sector de riego. Los 20 sensores soportados por el controlador, permite monitorear varias variables que tienen incidencia sobre las plantas, dentro de los sensores más destacados que soporta el dispositivo tenemos: temperatura y $\mathrm{RH} \%$ del aire, temperatura del suelo, VWC, CE y tensión del suelo. La transferencia de los datos del cultivo hasta el dispositivo ProFertin se realiza mediante comunicación inalámbrica o cableada y la configuración de los parámetros del controlador se realiza mediante una pantalla táctil a color de 7 " in situ o a través de una aplicación móvil desde cualquier parte del mundo con una conexión a internet.

Tabla 1. Parámetros de configuración del controlador

\begin{tabular}{lcc}
\hline \multicolumn{1}{c}{ Nombre } & Rango & Propósito \\
\hline Hora de encendido & $00: 00-23: 59$ & Fertirriego y General \\
Hora de apagado & $00: 00-23: 59$ & Fertirriego y General \\
Tiempo de activación & $>0$ (minuto o segundo) & Fertirriego y General \\
Tiempo de no activación & 0 (minuto o segundo) & Fertirriego y General \\
Valor mínimo asociado a la salida & $>0 \&<99999$ & Fertirriego y General \\
Valor máximo asociado a la salida & $>0 \&<99999$ & Fertirriego y General \\
Selección sensor asociado a la salida & $1-20$ & Fertirriego y General \\
Fertirrigación & ON - OFF & Fertirriego \\
Selección sensor de pH & $1-20$ & Fertirriego \\
\hline
\end{tabular}




\begin{tabular}{lcc}
\hline Selección sensor de CE & $1-20$ & Fertirriego \\
Motobomba & ON - OFF & Fertirriego \\
Control pH & ON - OFF & Fertirriego \\
Control CE & ON - OFF & Fertirriego \\
Control Humedad & ON - OFF & Fertirriego
\end{tabular}

Fuente de consulta: Manual técnico del controlador

ProFertin ya ha sido validado de forma simultánea con el proyecto de investigación "Evaluación de tres programas de fertilización edáfica en el cultivo de la cebolla de rama en la cuenca media del Río Otún", (Polanco Puerta \& Gómez Posada, 2017). Donde ProFertin fue utilizado como una de las metodologías de riego del diseño experimental de dicho proyecto.

\section{Sensores}

ProFertin recibe en tiempo real los datos de los sensores que toman información del suelo cerca de la raíz de la planta (VWC, CE, pH, temperatura) y del medio ambiente (temperatura y la humedad relativa del aire), información que es recolectada y procesada para ejecutar las acciones respectivas para realizar el control de cada uno de los parámetros que tienen incidencia en el desarrollo del cultivo. En el mercado existen muchos tipos de sensores que se utilizan en el sector agrícola, incluso cuando necesitamos controlar la humedad del suelo, encontramos diferentes tipos de sensores (Resistivos, Capacitivos, Tensiómetros) que hace que la elección del sensor sea un aspecto técnico importante a la hora de realizar la adquisición de los instrumentos de medida.

A continuación, se hace un análisis de los sensores utilizados en el proyecto de investigación "Desarrollo de un sistema controlado de fertirrigación y control ambiental" del CIAB.

Sensor de conductividad eléctrica: Este tipo de sensores mide la capacidad para conducir una corriente eléctrica a determinada temperatura y su valor se obtiene aplicando un voltaje entre dos electrodos y midiendo la resistencia de la solución, a mayor nivel de sales presentes en el suelo o en una solución, mayor es el valor de la 
conductividad, su valor se mide en deciSiemens / metro (dS/m) o milimhos / centímetro $(\mathrm{mmho} / \mathrm{cm})$ siendo:

$$
\frac{d s}{m}=\frac{m m h o}{c m}
$$

Este sensor es ampliamente utilizado para el control de niveles bajos o altos de fertilizante, ya que "la salinidad es un fenómeno indeseable que afecta el crecimiento de las plantas de varias maneras y por lo mismo, un aumento en la CE traerá como consecuencia una disminución de rendimiento" (Rebolledo, 2017, p.20).

Sensor de VWC: La medición de la humedad en el suelo se puede realizar de manera directa o indirecta, al ser implementado en sistemas de control automatizados en tiempo real, se hace necesario realizar una medición indirecta, puesto que para una medición directa es necesario tomar una muestra de suelo. Éste se obtiene al multiplicar la densidad aparente del suelo por el contenido gravimétrico de agua:

$$
\begin{aligned}
& \text { Contenido gravimétrico de agua }(\%)=\frac{\text { Peso del suelo húmedo }- \text { Peso del suelo seco } \times 100}{\text { Peso de suelo seco }} \\
& \text { Densidad aparente }=\frac{\text { Peso del suelo húmedo }- \text { Peso del suelo seco } \times 100}{\text { Peso de suelo seco }} \\
& \text { VWC }(\%)=\frac{\text { Densidad aparente del suelo }}{\text { densidad del agua }} \times \text { Contenido gravimétrico del agua }(\%)
\end{aligned}
$$

El sensor 5TE es un sensor multi parámetro que mide la temperatura, la CE y el VWC del suelo, todo en un mismo dispositivo (Figura 4). El dispositivo incorpora un termistor (Sensor de temperatura por resistencia) proporcionando la temperatura del suelo; los tornillos expuestos en el sensor forman una matriz eléctrica de 2 sensores para medir la conductividad eléctrica. 


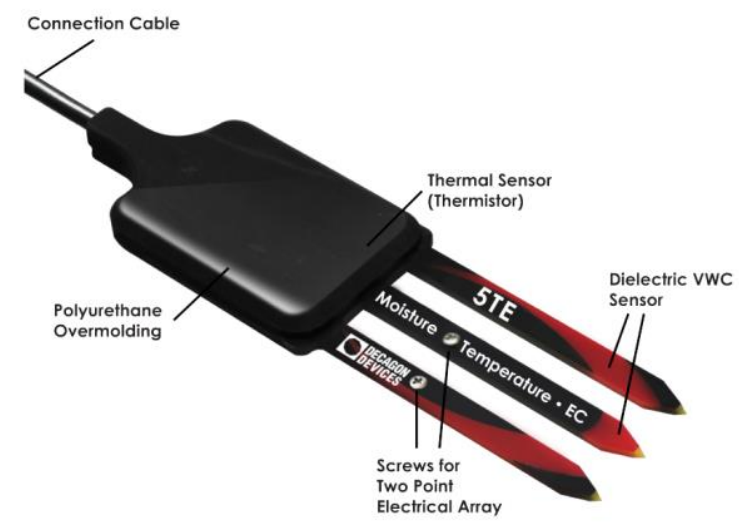

Figura 4. Componentes del Sensor de Conductividad Eléctrica (CE), Temperatura y Contenido Volumétrico de Agua (VWC)

El sensor calcula la Permisividad dieléctrica ( $\varepsilon a$ ) en un rango de 1 en aire a 80 en agua (tabla 2), existen numerosas ecuaciones de transferencia que permiten determinar el valor de VWC a partir de medidas de $\varepsilon a$. La calibración del sensor para suelos minerales se realiza mediante la siguiente ecuación:

$$
V W C=4.3 \times 10^{-6} \varepsilon_{a}^{3}-5.5 \times 10^{-4} \varepsilon_{a}^{2}+2.92 \times 10^{-2} \varepsilon_{\mathrm{a}}-5.3 \times 10^{-2}
$$

Tabla 2. Especificaciones técnicas del sensor 5TE

\begin{tabular}{|c|c|c|c|}
\hline Parámetro & Rango & Resolución & Precisión \\
\hline Temperatura & -40 to $60 \cdot \mathrm{C}$ & $0.1 \circ \mathrm{C}$ & $\pm 1 \circ \mathrm{C}$ \\
\hline CE & $\begin{array}{l}0 \text { to } 23 \mathrm{dS} / \mathrm{m} \\
\text { (bulk) }\end{array}$ & $\begin{array}{c}0.01 \mathrm{dS} / \mathrm{m} \text { para } 0 \text { to } 7 \\
\mathrm{dS} / \mathrm{m}, 0.05 \mathrm{dS} / \mathrm{m} \text { para } 7 \text { to } \\
23 \mathrm{dS} / \mathrm{m}\end{array}$ & $\begin{array}{c} \pm 10 \% \text { para } 0 \text { to } 7 \mathrm{dS} / \mathrm{m}, \\
\text { requiere calibración para } \\
\text { valores }>7 \mathrm{dS} / \mathrm{m} .\end{array}$ \\
\hline vwc & $\begin{array}{c}\text { Permisividad } \\
\text { dieléctrica } \\
\text { aparente }\left(\varepsilon_{a}\right): 1 \\
\text { (aire) a } 80 \text { (agua) }\end{array}$ & $\begin{array}{c}\text { Resolución: } \varepsilon_{a}: 0.1 \varepsilon_{a} \\
\text { (sin unidad) de } 1 \text { a } 20, \\
<0.75 \varepsilon_{a} \text { (sin unidad) de } \\
20 \text { a } 80 \text { VWC: } 0.0008 \mathrm{~m} 3 / \\
\text { m3 }(0.08 \% \text { VWC) de } 0 \text { a } \\
50 \% \text { VWC }\end{array}$ & $\begin{array}{l}\text { Usando la ecuación de } \\
\text { Topp: } \pm 0.03 \mathrm{~m} 3 / \mathrm{m} 3( \pm \\
3 \% \text { VWC) típico En suelos } \\
\text { minerales que tienen } \\
\text { solución conductividad } \\
\text { eléctrica. }<10 \mathrm{dS} / \mathrm{m}\end{array}$ \\
\hline
\end{tabular}

Fuente de consulta: Manual técnico del sensor

\section{Protocolos de comunicación}

Para la instalación de los sensores en campo el prototipo ProFertin soporta dos métodos de comunicación que permiten adaptarse fácilmente a las condiciones topográficas y específicas de cada cultivo. El estándar de comunicación industrial RS485 es el primer método soportado por el 
controlador, este es una comunicación cableada que puede transmitir datos a altas velocidades y a largas distancias (10 Mbit/s hasta 12 metros y $100 \mathrm{kbit} / \mathrm{s}$ en 1200 metros) y permite la comunicación de múltiples sensores a través de un mismo cable par trenzado (Figura 5). "El estándar industrial RS485 o también conocido como EIA-485 es un estándar de capa física según el modelo OSI. Es decir, no pone normas ni restricciones sobre el contenido, forma, o codificación de los mensajes enviados" (Naylampmechatronics, 2015, p.1). Este es el método más accesible para los pequeños y medianos agricultores debido al bajo costo de su implementación en áreas no muy extensas de cultivo.

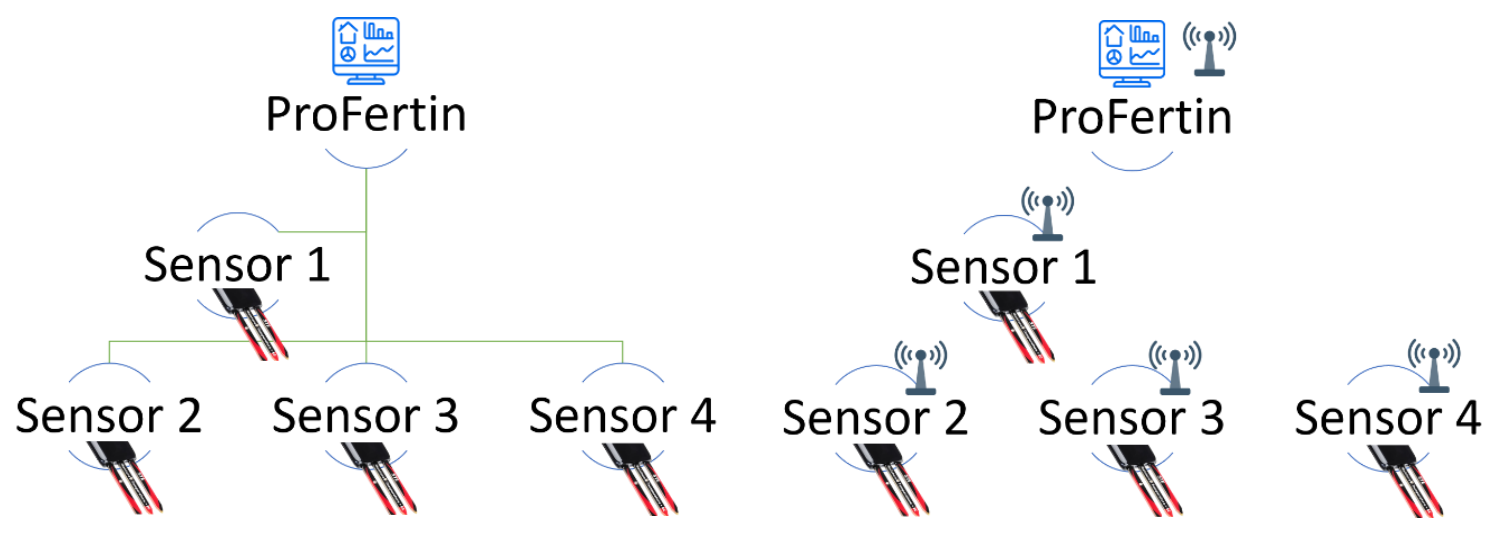

Figura 5. a) Red de comunicación cableada, b) Red de comunicación inalámbrica

La comunicación inalámbrica es otro método utilizado para enviar la información de los sensores instalados en campo hasta ProFertin. Los sensores que implementan esta comunicación pueden tener un alcance de hasta $9.6 \mathrm{~km}$ con antena dipolo y un alcance de hasta $24 \mathrm{~km}$ con antena de alta ganancia en condiciones ideales, sin necesidad de contratar un proveedor de servicio de internet. Un valor agregado de estos módulos de sensores inalámbricos, es la implementación de energía solar como fuente de alimentación, con una autonomía de funcionamiento de 1 semana sin recibir carga solar gracias a los algoritmos utilizados para minimizar el consumo de energía de los dispositivos electrónicos y de comunicación. 


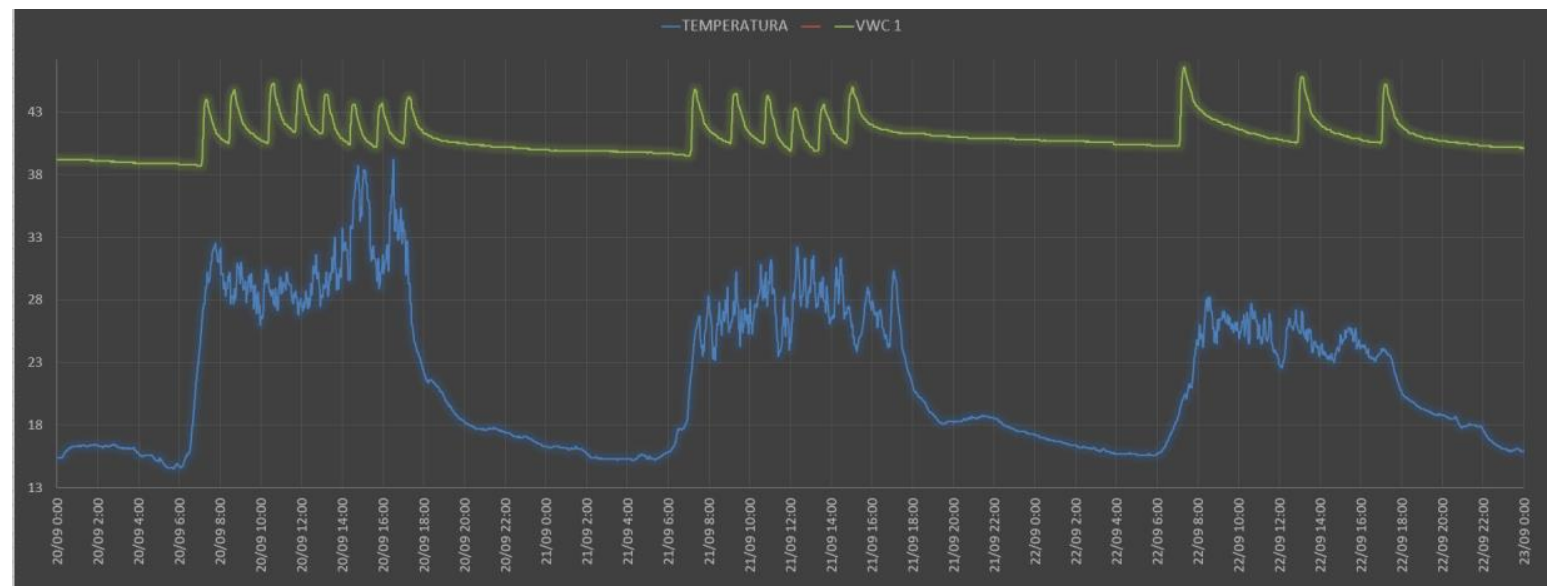

Figura 6. Comportamiento de la humedad en el suelo de un sistema automatizado

En la figura 5, se observa el comportamiento de la humedad en el suelo durante tres días con un comportamiento diferente de temperatura (Gráfica color azul); en los días de mayor temperatura se registra un incremento de las aplicaciones de agua, cada pico en el comportamiento de la humedad (Gráfica color azul) es el indicador de aplicación de agua realizada por el sistema automatizado.

\section{CONCLUSIONES.}

Las nuevas tecnologías aplicadas en el sector agrícola permiten realizar un uso más eficiente de agua e insumos agrícolas, logrando un ahorro significativo de agua con respecto al método tradicional utilizado por el agricultor; un ahorro en el consumo de agua que se ve reflejado en la productividad del cultivo y contribuye a mitigar los efectos negativos producidos por el cambio climático. La transferencia de tecnologías del sector agrícola para los pequeños y medianos agricultores, contribuye a minimizar la brecha tecnológica que existe entre los grandes productores y los campesinos colombianos; la implementación de estas tecnologías permite que los productores tengan mayor rentabilidad de sus predios, por el incremento en la productividad, ahorro energético, menor consumo de agua y fertilizantes, y reducción en los costos de mano de obra derivada del manejo del sistema de riego tradicional.

\section{BIBLIOGRAFÍA.}

Corredor Camargo, E., Fonseca Carreño, J., \& Páez Barón, E. (2012). Los servicios ecosistémicos de regulación: tendencias e impacto en el 
bienestar humano. Revista de Investigación Agraria y Ambiental, 3(1), 77-83. doi: https://doi.org/10.22490/21456453.936

Hidro Norte. (2018). Ingenieria de sistemas de riego por goteo. Recuperado de http://chile.hidronorte.cl/servicios/ingenieriaconstruccion/ingenieria-sistema-goteo-riego/

Liotta, M., Carrión, R., Ciancaglini, N., \& Olguín, A. (2015). Manual de capacitación: Riego por goteo. PROSAP. Recuperado de https://inta.gob.ar/sites/default/files/inta_manual_riego_por_goteo.pdf

Mendoza Velásquez, S., Cano Muñoz, J., \& Rojas Sánchez, F. (2015). Acción comunitaria frente al fenómeno del cambio climático, en el páramo de la región del Guavio, Cundinamarca, Colombia. Revista de Investigación Agraria y Ambiental, 6(1), 265 - 279. doi: https://doi.org/10.22490/21456453.1286

Montenegro Gómez, S., \& Hernández Ossa, Y. (2015). Biotecnología aplicada al desarrollo agropecuario colombiano. Revista de Investigación Agraria y Ambiental, 6(2), 97 - 108. doi: https://doi.org/10.22490/21456453.1408

Naylampmechatronics. (2015). Comunicación RS485 con Arduino. Recuperado de https://naylampmechatronics.com/blog/37_Comunicaci\%C3\%B3nRS485-con-Arduino.html

Polanco Puerta, M. F., \& Gómez Posada, S. (2017). Evaluación de tres programas de fertilización edáfica en el cultivo de la cebolla de rama en la cuenca media del río Otún. Intropica, 33. Recuperado de http://dx.doi.org/10.21676/23897864.2203

Rebolledo, S. (2017). Conductividad eléctrica y salinidad. Redagrícola. Recuperado de http://www.redagricola.com/cl/conductividad-electricasalinidad/

Zamora Cardona, J., \& Cristancho, F. (2008). La Humedad en las Propiedades Físicas del Suelo. Recuperado de http://gfnun.unal.edu.co/fileadmin/content/gruposdeinvestigacion/fisica nuclear/Tesis/JCZamoraTG08.pdf 